\title{
THE SECOND BIRKHOFF THEOREM FOR OPTICAL HAMILTONIAN SYSTEMS
}

\author{
LEONID POLTEROVICH
}

(Communicated by Kenneth R. Meyer)

\begin{abstract}
Consider a smooth Hamiltonian function on the cotangent bundle of the $n$-dimensional torus such that its restriction on every fiber is strictly convex. Let $L$ be a Lagrange invariant torus of the Hamiltonian flow which is homologous to the zero section. We show that, under some assumptions, $L$ is a smooth section of the cotangent bundle.
\end{abstract}

\section{INTRODUCTION AND MAIN RESULTS}

In [B], G. D. Birkhoff developed the qualitative theory of symplectic twist maps of the cylinder $C=\mathbf{R}^{1} \times \mathbf{S}^{1}$ (see [H1] for a modern survey). One of his theorems (which is known as the second Birkhoff theorem) states that any embedded noncontractible invariant circle of such a symplectomorphism is a section of the R-bundle $C$ over $\mathbf{S}^{1}$. In the present paper we prove the analogous assertion for Lagrange invariant tori of optical Hamiltonian systems on the cotangent bundle $T^{*} \mathbf{T}^{n}$ of the $n$-dimensional torus.

Definition. A smooth Hamiltonian function $H: T^{*} \mathbf{T}^{n} \rightarrow \mathbf{R}$ is called optical if its restriction to every fiber of the cotangent bundle is strictly convex (i.e., $H_{p p}>0$, where $p$ is a linear coordinate on a fiber).

Example. The Hamiltonian function associated with a Riemannian metric on the torus is optical.

Main Theorem. Let $L \subset T^{*} \mathbf{T}^{n}$ be a smooth Lagrange torus which lies in a regular level $\{H=$ const $\}$ of an optical Hamiltonian function. Assume that

(c1) $L$ is homologous to the zero section;

(c2) the Hamiltonian flow on $L$ preserves a smooth measure.

Then $L$ is a smooth section of the cotangent bundle.

Remarks.

1. For the case $n=2$, this theorem (under slightly different assumptions) was proved together with $\mathrm{M}$. Bialy in [BP1] (see also [Bi], [Po]). In the present work we use to a great extent the ideas of [BP1].

Received by the editors November 11, 1989.

1980 Mathematics Subject Classification (1985 Revision). Primary 58F05; Secondary 53C57. 
2. In [A1], V. I. Arnold suggested a program of studying topological properties of optical Lagrange submanifolds. This program includes the hypothesis that (under some additional assumptions) such a submanifold is a section of the cotangent bundle.

3. One can find some interesting generalizations of Birkhoff's theory to the $n$-dimensional case in an article by M. Herman [H2].

4. The following result is crucial for our proof:

Proposition [Vi], [LS]. The Maslov class of any embedded Lagrange torus in $T^{*} \mathbf{T}^{n}$ which is homologous to the zero section is zero.

The proof of this proposition is based on the result by C. Viterbo [Vi] concerning the Maslov class of embedded Lagrange tori in $\mathbf{C}^{n}$.

5. It seems that the assertion of the theorem holds without the measurepreserving condition (c2). However, this condition is rather natural for Hamiltonian systems of classical mechanics. For example, it holds for Lagrange invariant tori carrying quasi-periodic motion.

The following corollary is a simple generalization of [BP2, Theorem 1a], see also [BP1].

Corollary 1. Consider a smooth Lagrange invariant torus of the Hamiltonian system associated with a Riemannian metric on $\mathbf{T}^{n}$. Assume that the conditions (c1) and (c2) of the main theorem hold. Then the natural projection of any trajectory of the Hamiltonian flow which lies on this torus is a minimal geodesic (this means that a lifting of such a geodesic to the covering space $\mathbf{R}^{n}$ minimizes the Riemannian distance between any two of its points).

Corollary 2. Let $d$ be a Riemannian metric on $\mathbf{T}^{n}$ which has a "bump" in the following sense: There exists an embedded ball $B^{n} \subset \mathbf{T}^{n}$ and a point $K \in B$ such that $2 d(K, \partial B)>\operatorname{diam}(\partial B)$, where $\operatorname{diam}(\partial B)$ is the diameter of $\partial B$ in the induced Riemannian metric on $\partial B$. Then the geodesic flow associated with the metric $d$ has no Lagrange invariant tori satisfying the conditions ( $\mathrm{c} 1)$ and (c2) of the main theorem.

Proof of Corollary 2. Due to Corollary 1, it is sufficient to show that no minimal geodesic of the metric $d$ passes through the point $K$. Suppose that such a minimal geodesic exists. Then it contains a segment $g$ such that $K \in g \subset$ $B$ and its ends lie on the boundary $\partial B$. Thus $2 d(K, \partial B) \leq$ length $(g) \leq$ $\operatorname{diam}(\partial B)$. This inequality contradicts our assumptions.

Remark 6. An analogous example of a Riemannian metric with a "bump" on a two-dimensional torus was constructed in [BP1], [Ba], and [BR2].

\section{Proof of The MAIN THEOREM}

1. Denote by $\pi: T^{*} \mathbf{T}^{n} \rightarrow \mathbf{T}^{n}$ the natural projection. Define the critical set $S(W)$ of a closed Lagrange submanifold $W$ as the set of critical points of the 
restriction $\left.\pi\right|_{W}$. We will say that $W$ is generic if $S$ consists of an $(n-1)$ dimensional submanifold $S^{0}$ and its boundary $S \backslash S^{0}$ whose codimension in $W$ is not less than 3 .

2. We claim that it is sufficient to prove the theorem for Lagrange tori which are generic. It is well known [A2, Appendix 12] that there exists a sequence $F_{k}$ of symplectomorphisms of the cotangent bundle such that $\left\{F_{k}\right\} C^{\infty}$-converges to the identity and all $F_{k}(L)$ are generic.

It is clear that, for large $k$, all Lagrange tori $F_{k}(L)$ satisfy the conditions of the theorem with the optical Hamiltonian function $H_{k}=H \circ F_{k}^{-1}$. Due to our assumption, each $F_{k}(L)$ is the graph of a smooth map $\Phi_{k}: \mathbf{T}^{n} \rightarrow \mathbf{R}^{n}$ (we identify $T^{*} \mathbf{T}^{n}$ with $\mathbf{T}^{n} \times \mathbf{R}^{n}$ ).

It is easy to check that the property of $H_{k}$ of being optical implies that the corresponding Hamiltonian field is monotone positive in the sense of $\mathbf{M}$. Herman [H2, p. 15]. Thus all the maps $\Phi_{k}$ satisfy the a priori Lipschitz estimate $\left\|D \Phi_{k}\right\|_{L_{\infty}}<C$, where $C$ depends only on $H$ and the norm of the perturbation $\| F_{k}-$ id $\|_{C^{\infty}}$. These estimates and the smoothness of $L$ give us the result that $L$ is the graph of some smooth map $\mathbf{T}^{n} \rightarrow \mathbf{R}^{n}$. Our claim is proved.

3. In the following we will assume that $L$ is generic. Suppose that the critical set $S$ is nonempty.

Lemma $1[\mathrm{Ch}]$. The Hamiltonian field $v$ is not tangent to the critical set $S$.

Thus the Hamiltonian field determines a coorientation (and the orientation) of the cycle $S$.

Lemma 2. The cycle $[S]$ is dual to the Maslov class of the Lagrange torus $L$.

This lemma is a simple generalization of Theorem 2.1a of [BP1]. To prove it, it is sufficient to check that the Hamiltonian field in the nonsingular points of $S$ is directed to the positive side with respect to the canonical Maslov coorientation. The proof is based on the fact that $H$ is optical.

Lemma 3. The cycle $S$ is homologous to zero: $[S]=0$.

Proof. The Maslov class of the Lagrange torus $L$ is zero (see Remark 4). The statement of the lemma follows now from Lemma 2.

Thus there exists an $n$-dimensional chain $U$ such that $\partial U=S$. Denote by $\Omega$ the volume form which is preserved by the Hamiltonian field $v$. This means that $d i_{v} \Omega=0$. From Lemma 1 one can conclude that $\int_{S} i_{v} \Omega \neq 0$. On the other hand, $\int_{S} i_{v} \Omega=\int_{U} d i_{v} \Omega=0$. This contradiction proves the theorem.

\section{ACKNOWLEDGMENTS}

I am deeply grateful to V. I. Arnold for useful discussions and to J. -C. Sikorav who acquainted me with the result mentioned in Remark 4. 


\section{REFERENCES}

[A1] V. I. Arnold, First steps in symplectic topology, Russian Math. Surv. 41 (1986), 1-21.

[A2] _ Mathematical methods of classical mechanics, Berlin, Heidelberg, New York, 1978.

[B] G. D. Birkhoff, Surface transformations and their dynamical applications, Acta Math. 43 (1920), 1-119; Collected Math. Papers, vol. 2, pp. 119-229.

[Ba] V. Bangert, Mather sets for twist maps and geodesics on tori, Dynamics Reported 1 (1987),

[Bi] M. L. Bialy, Aubry-Mather sets and Birkhoff's theorem for geodesic flows on the two-dimensional torus, Comm. Math. Phys. 126 (1989), 13-24.

[BP1] M. L. Bialy and L. V. Polterovich, Lagrangian singularities of invariant tori of Hamiltonian systems with two degrees of freedom, Invent. Math. 97 (1989), 291-303.

[BP2] __ Geodesic flows on the two-dimensional torus and phase transitions "commensurabilityincommensurability," Funct. Anal. Appl. 20 (1986), 260-266,

[Ch] Yu. V. Chekanov, Caustics in geometric optics, Funct. Anal. Appl. 20 (1986), 223-226.

[H1] M. Herman, Sur les courbes invariantes par les diffeomorphismes de l'anneau, Asterisque 103-104 (1983).

[H2] _ Existence et non existence de tores invariants par des diffeomorphismes symplectiques, Ecole Polytechnique, Centre de Mathematiques, Equations aux derivees partielles (Seminaire 1987-1988), Expose 14 (1988).

[LS] F. Lalonde and J.-C. Sikorav, Sous-varietes lagrangiennes exactes des fibres cotangents, Comment. Math. Helv. (to appear).

[Po] L. V. Polterovich, The Maslov class of the Lagrange surfaces and Gromov's pseudo-holomorphic curves, Trans Amer. Math. Soc. (to appear).

[Vi] C. Viterbo, A new obstruction to embedding Lagrangian tori, Invent. Math. 100 (1990), 301-320.

107392, Halturinskaja 4-2-60, Moscow, USSR afFiliation

Current address: School of Mathematical Sciences, Tel-Aviv University, Ramat-Aviv, 69978 Tel-Aviv, Israel 